\title{
Long non-coding RNA00887 reduces the invasion and metastasis of non-small cell lung cancer by causing the degradation of miRNAs
}

\author{
YINGXUAN TIAN ${ }^{1,2}$, MIN YU $^{3}$, LI SUN $^{2}$, LINGHUA LIU $^{2}$, SHUFEN HUO $^{2}$, WENLI SHANG $^{2}$, \\ SEN SHENG ${ }^{4}$, JUN WANG ${ }^{2}$, JINGYING SUN ${ }^{5}$, QIAOXIA HU ${ }^{5}$, YAWEI DOU ${ }^{6}$, \\ JIANFEI ZHU ${ }^{6}$, XIAOPING REN ${ }^{2}$ and SHUANYING YANG ${ }^{1}$
}

\footnotetext{
${ }^{1}$ Department of Respiratory Medicine, The Second Affiliated Hospital, Xi'an Jiaotong University, Xi'an, Shaanxi 710004; Departments of ${ }^{2}$ Elderly Respiratory Medicine and ${ }^{3}$ Oncology Medicine, Shaanxi Provincial People's Hospital, Xi'an, Shaanxi 710068, P.R. China; ${ }^{4}$ Neurology Department, University of Arkansas for Medical Science, Little Rock, AR 72205, USA; ${ }^{5}$ Central Laboratory; ${ }^{6}$ Department of Thoracic Surgery, Shaanxi Provincial People's Hospital, Xi'an, Shaanxi 710068, P.R. China
}

Received August 31, 2018; Accepted July 5, 2019

DOI: $10.3892 / o r .2019 .7228$

\begin{abstract}
Long non-coding RNAs (lncRNAs) can act as carcinogenic or cancer suppressive factors during the pathogenesis, invasion and metastasis of non-small cell lung cancer (NSCLC). The current study explored the role of long intergenic non-protein coding RNA 00887 (LINC00887) and competing endogenous RNAs (ceRNAs). It was revealed that LINC00887 interacts with several microRNAs (miRs), which regulates downstream genes such as fibronectin 1, MET proto-oncogene, receptor tyrosine kinase and mothers against decapentaplegic homolog 4, which are associated with the spread of lung cancer. The experimental results also suggested that LINC00887 can stimulate miR-613, miR-206 and miR-1-2 to become competing endogenous RNAs, which may regulate the epithelial-mesenchymal transition of NSCLC cells through the transforming growth factor-â signal transduction pathway, and therefore promote the migration of cells and the acquisition of stem cell characteristics. Therefore, it can be concluded that high levels of LINC00887 can accelerate the malignant transformation ability of NSCLC cells.
\end{abstract}

\section{Introduction}

Global cancer statistics report that lung cancer has been the primary cause of cancer-associated mortality in males and females in the past two decades $(1,2)$. In China, lung cancer

Correspondence to: Dr Shuanying Yang, Department of Respiratory Medicine, The Second Affiliated Hospital, Xi'an Jiaotong University, 36 Xiwu Road Xi'an, Shaanxi 710004, P.R. China

E-mail: yangshuanying66@163.com

Key words: long non-coding RNA, linc00887, lung cancer, metastasis, competing endogenous RNAs mortality remains high and is likely to continue to rise (3). Currently, genetic and genomic methods are important tools, and have been utilized for investigating non-small cell lung cancer (NSCLC) pathogenesis, diagnosis and treatment (4-6).

Long non-coding RNAs (lncRNAs) are RNA molecules of typically $>200$ nucleotides, but with no protein-coding capability (7). It has been reported that some lncRNAs can act as carcinogenic or cancer-suppressive factors associated with tumour cell invasion and metastasis in NSCLC, including HOX transcript antisense RNA (8), prostate cancer associated transcript 1 (9), PCGEM1 prostate-specific transcript (10) and transcribed ultra-conserved region 338 (11). On the contrary, various other lncRNAs, such as growth arrest specific 5 (12), tumour protein p53 pathway corepressor 1 (13), maternally expressed 3 (14) and phosphatase and tensin homolog pseudogene 1 (15), can reduce the metastasis and invasion of tumour cells through suppressive actions. Therefore, the role of lncRNAs in tumours is complex and diverse. Additionally, the signalling pathways that lncRNAs act on are also varied, including the phosphoinositide 3-kinase/protein kinase $\mathrm{B}$ and transforming growth factor- $\beta$ (TGF- $\beta$ )/SMAD3 pathways $(16,17)$. Certain types of 1 ncRNA can be used as biomarkers for clinical diagnosis and prognosis assessment, including metastasis associated lung adenocarcinoma transcript 1 (18). Competing endogenous RNAs (ceRNAs) are subset of lncRNAs that have an important role in cell cycle progression; however, there are only a few reports available in the literature regarding their role in tumourigenesis (19).

In this study, a lncRNA array was used to screen a group of lncRNAs associated with lung cancer metastasis. The role LINC00887 and its associated ceRNA in NSCLC invasion and metastasis were systematically investigated. Lung cancer cell lines, 95-C and 95-D were cultured and analysed with respect to LINC00887 and its associated microRNAs (miRNAs/miRs). Subsequently, the proliferation, apoptosis and migration of lung cancer cells, and role of LINC00887 were examined. 


\section{Materials and methods}

Cell culture and RNA extraction. The 95-C cell line is a low metastatic ability human lung cancer cell, and 95-D is a high metastatic cell line, both of which were purchased from the Cell Bank of the Chinese Academy of Sciences (Shanghai, China). RPMI-1640 (Thermo Fisher Scientific, Inc., Waltham, MA, USA) medium containing $10 \%$ foetal bovine serum (FBS; Gibco; Thermo Fisher Scientific, Inc.) and 1\% dual antibiotic (chloramphenicol and streptomycin) was used for cell culture at $37^{\circ} \mathrm{C}$ and $5 \% \mathrm{CO}_{2}$ in a saturated humidity incubator. The cells were cultured at a $10 \times 10^{9} / \mathrm{ml}$ density, and then harvested for RNA extraction.

Total RNA was extracted from cells using TRIzol reagent (Invitrogen; Thermo Fisher Scientific, Inc.), following the manufacturer's instructions. The homogenate samples were incubated at $15-30^{\circ} \mathrm{C}$ for $5 \mathrm{~min}$ to completely dissociate the nucleoprotein complex. For every $1 \mathrm{ml}$ TRIzol reagent, $0.2 \mathrm{ml}$ chloroform was added, and the samples were shaken vigorously for $15 \mathrm{sec}$, then incubated at $20^{\circ} \mathrm{C}$ for $3 \mathrm{~min}$. The samples at were centrifuged at $4^{\circ} \mathrm{C}$ and $12,000 \mathrm{x}$ g for $15 \mathrm{~min}$. RNA was in the upper aqueous phase, and the volume of the aqueous phase was $\sim 60 \%$ of the total volume of TRIzol reagent used. After incubation at $20^{\circ} \mathrm{C}$ for $10 \mathrm{~min}$, the samples underwent centrifugation at $4^{\circ} \mathrm{C}$ and $12,000 \mathrm{xg}$ for $10 \mathrm{~min}$. The RNA solution obtained was stored at $-70^{\circ} \mathrm{C}$. Each sample was quantified, and the purity was determined by the absorbance ratio at 260 and $280 \mathrm{~nm}$ (A260/A280).

LncRNA array analysis. Total RNA from 95-C and 95-D cells were analysed using the GeneSpring GX v12.0 software (Agilent Technologies, Inc., Santa Clara, CA, USA) and lncRNA microarray (Human LncRNA Array v3.0; Arraystar, Inc., Rockville, MD, USA), and scanned with the Agilent G2505C scanner (Agilent Technologies, Inc.). Differentially expressed lncRNAs were selected by fold change filtering (fold change $\geq 2.0$ ), and then, for those that were statistically significant, volcano plot filtering (fold change $\geq 2.0, \mathrm{P} \leq 0.05$ ). Subsequently, LINC00887 was selected with the highest statistical score and validated as the research object of the study. The datasets generated and/or analysed during the current study are available in the Gene Expression Omnibus repository (ncbi.nlm.nih.gov/geo/query/acc.cgi?acc=GSE124619).

Bioinformatic analysis of LINC00887 and reverse transcription-quantitative polymerase chain reaction (RT-qPCR) validation. Bioinformatic analysis of LINC00887 was performed using the Ensembl (asia.ensembl.org) and eBioPortal (cbioportal.org/) databases. PrimeScript ${ }^{\circledR}$ First Strand cDNA Synthesis Kit to perform RT. TaqMan ${ }^{\circledR}$ MicroRNA Reverse Transcription Kit was used for microRNA RT, with a incubation at $30^{\circ} \mathrm{C}$ for $10 \mathrm{~min}$ and $42^{\circ} \mathrm{C}$ and $30 \mathrm{~min}$. PCR was conducted using SYBR Select Master Mix (Thermo Fisher Scientific, Inc.) and a 7500 Fast Real-Time PCR system at $50^{\circ} \mathrm{C}$ for $2 \mathrm{~min}$ and $95^{\circ} \mathrm{C}$ for $10 \mathrm{~min}$, followed by cycles of $95^{\circ} \mathrm{C}$ for $15 \mathrm{sec}$ and $60^{\circ} \mathrm{C}$ for $1 \mathrm{~min}$. LINC00887 primers were as follows: Forward, 5'-TGGCCAGTGTTTCACCTGTT-3' and reverse, 3 '-TGATTTCCTCCAACGTGCCA-5'. $\beta$-actin primers were as follows: Forward, 5'-ATCCAGGCTGTGCTATCCCT-3' and

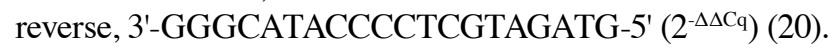

RNA interference and cell proliferation. The types of LINC00887 small interfering RNA sequences (siRNA; 5'-GGCCTTTGCAGTTATTAGGAA-3' and control RNA sequence 5'-GGCCTTTGCGTCACGCCTTAG-3'; Guangzhou RiboBio Co., Ltd., Guangzhou, China) were synthesized and the suppressive effect in 95-D cells was analysed by RT-qPCR. siRNA2 was transfected into 95-D cell lines. Opti-MEM (Gibco; Thermo Fisher Scientific, Inc.) was added when the cell confluence reached 70-80\%. 95-C-pGL3 vector, 95-C-LINC00887, 95-D-control and 95-D-siRNA were transfected ( $2 \mu \mathrm{g}$ plasmid or 100 pmol siRNA per well) using Lipofectamine ${ }^{\circledR} 3000$ for $8 \mathrm{~h}$, and then continuously cultured for $24 \mathrm{~h}$. The cell number in each group was controlled at $\sim 3 \times 10^{4} / \mathrm{ml}$. Each well was topped up with $50 \mu 1$ MTT solution and maintained for $4 \mathrm{~h}$. After $4 \mathrm{~h}$, the culture solution in the 96-well plate was aspirated with a pipette. Dimethyl sulfoxide $(150 \mu \mathrm{l})$ was added to each well, and the culture plate was placed on a microplate reader. The setup procedure involved shaking for $5 \mathrm{~min}$ and optical density (OD) was recorded at a wavelength of $490 \mathrm{~nm}$. The tumour cell growth inhibition rate (IR) was calculated using the formula: Cell growth IR $(\%)=[1-(\mathrm{OD}$ value of experimental group-OD value of blank groups)/(OD value of control group-OD value of blank groups) $\mathrm{x} 100$.

Detection of cell invasion and migration ability. Cells were cultured and transfected for $48 \mathrm{~h}$, then a cell suspension $\left(1 \times 10^{6}\right.$ cells $\left./ \mathrm{ml}\right)$ was produced in $1 \mathrm{X}$ binding buffer. The cell cycle was analysed in flow cytometer with an Annexin V-fluorescein isothiocyanate (FITC) and Annexin V-propidium iodide (PI) reagent kit (BestBio Science). Subsequently, the 95-C cell concentration was reduced to $3 \times 10^{4} \mathrm{cell} / \mathrm{ml}$ and a Transwell assay was performed using Millipore chambers (8- $\mu \mathrm{m}$ diameter). DMEM (500 $\mu \mathrm{l})$ without FBS was used to dilute the Matrigel (BD Biosciences; Becton-Dickinson and Company, Franklin Lakes, NJ, USA; 1:3) and spread on the upper chambers (10\% FBS in DMEM; $50 \mu 1 /$ well). DMEM solution containing 10\% FBS (HyClone; GE Healthcare Life Sciences, Logan, UT, USA) was used to dilute the fibronectin $(1: 2,000)$ and $50 \mu \mathrm{l} /$ well was added to lower chambers. The above reagents were also applied to the 95-D cell chambers. Cells in both chambers were cultured for a further $24 \mathrm{~h}$. Finally, the remaining cells were counted under a microscope after fixing in $75 \%$ methanol and staining $\left(3 \mathrm{~min}, 25^{\circ} \mathrm{C}\right)$.

In vitro tumour sphere formation assays. 95-D cells $\left(1 \times 10^{6} / \mathrm{ml}\right)$ were cultured in 6-well dishes. Tumour sphere nutrient solution was prepared using a formula consisting of $500 \mathrm{ml} \mathrm{DMEM} / \mathrm{F} 12,20 \mathrm{ng} / \mathrm{ml}$ epidermal growth factor, $10 \mathrm{ng} / \mathrm{ml}$ basic fibroblast growth factor and $5 \mu \mathrm{g}$ insulin, and diluted with B27 supplement at 1:50. Samples $(20 \mu \mathrm{l})$ of each group were collected $\left(3 \times 10^{4}\right.$ cells $\left./ \mathrm{ml}\right)$ and stained with trypan blue $(0.4 \%, 5 \mathrm{~min})$. Cellular morphology was observed using a bright field microscope, at a diluted concentration of one cell per $\mu 1$.

Construction of the overexpression plasmid and LINC00887 promoter reporter. The LINC00887 gene was inserted into pcDNA3.1+ vector and LINC00887 enhancer into the 
pGL3 basic vector. Takara reverse transcription kit (Takara Biotechnology Co., Ltd., Dalian, China) was used to prepare the LINC DNA from lung adenocarcinoma tissue specimens. The LINC00887 gene was amplified by PCR, 2X Taq MasterMix (Tiangen Biotech Co., Ltd., Beijing, China; $\mathrm{Tm}$ at $\left.66-65^{\circ} \mathrm{C}\right)$. The restriction endonuclease was used to hydrolyze the product and vector of PCR [pcDNA3.1 (+)], and 95-D cell genome was extracted using the QIAamp DNA Mini Kit (Qiagen, Inc., Valencia, CA, USA). The LINC00887 gene does not have specific promoter, but an enhancer was found in the Ensembl database (http://useast. ensembl.org/Homo_sapiens/Gene/Summary?db=core;g=EN SG00000214145;r=3:194296465-194312803). Online analysis in the Ensembl database indicated that the enhancer sequence contains a TPA-responsive element binding sequence [TGA $(\mathrm{C} / \mathrm{G}) \mathrm{TCA}]$ and SMAD binding element binding motifs (GTCT/AGAC). The enhancer sequence was amplified by Q5 PCR polymerase chain reaction. The recombinants and PRL-TK plasmids were transformed into DH5 $\alpha$ competent cells. Plasmid Mini Kit (Omega Bio-Tek, Inc., Norcross, GA, USA) was used to extract plasmids and they were identified by electrophoresis and sequencing.

LINC00887 intracellular location and TGF- $\beta$ signal pathway regulation. $95-\mathrm{C}$ and $95-\mathrm{D}$ cells $\left(1 \times 10^{6}\right.$ cells $\left./ \mathrm{ml}\right)$ were cultured on glass slides, and an 18S probe (Guangzhou RiboBio Co., Ltd., Guangzhou, China) was prepared for fluorescence in situ hybridization. A laser scanning confocal microscope (LSCM; OLYMPUS Fluoview FV1000; Olympus Corporation, Tokyo, Japan) was used to observe and locate LINC00887 in 95-C and 95-D cells. The cells were initially cultured in Opti-MEM solution with Lipofectamine ${ }^{\circledR} 3000$ transfection reagent for $48 \mathrm{~h}$, then continuously cultured for a further $48 \mathrm{~h}$ with $10 \mu \mathrm{g} / \mathrm{ml}$ TGF- $\beta$ and $5 \mu \mathrm{m}$ ITD- 1 . When analysing the TGF- $\beta$ pathway, pcDNA3.1-LINC00887 plasmid was also transfected for $48 \mathrm{~h}$ in the indicated groups ( $2 \mu \mathrm{g}$ /well in 6-well plates). Luciferase Assay System (Promega Corporation, Madison, WI, USA) and luminometer (Hitachi, Ltd., Tokyo, Japan) was used to examine the association between TGF- $\beta$ and LINC00887 expression. Cells were transfected with pGL3 basic vector (Firefly luciferase) and pRL-TK plasmid (Renilla luciferase). LINC promoter was synthesized and insert into pGL3 basic and the empty vector was used as control.

Prediction of LINC00887-targeting miRNAs and validation. LNCipedia (Incipedia.org/db/search) database was used to predict and screen the miRNAs with mutual downstream genes. miRNA precursors (CCTCGAGCTATTCTCATTCATTATG XhoI) and pGL3 empty vector or pGL3-LINC00887 were transfected into 293T cells (Cell Bank of the Chinese Academy of Sciences, Shanghai, China) for $24 \mathrm{~h}$, and then tested for luciferase activity.

miRNA expression and regulation by LINC00887. Three LINC00887 siRNAs were synthesized (Guangzhou RiboBio Co., Ltd.) and the suppression effect of Linc 00887 on 95-D cells was analysed by RT-qPCR. LINC00887 was overexpressed in the 95-C cell line using the pcDNA3.1-LINC0088 vector. siRNA2 was transfected into 95-D cell lines, and the expression levels of miRNAs were detected using a TaqMan probe.
The argonaute-2 (Ago2) antibody was sequentially processed for immunoprecipitation, nuclei acid purification and quantification. The details of the experiment are as follows: 95-D cells were digested and collected in Eppendorf tubes, washed in PBS, and the Magna RIP RNA-Protein Immunoprecipitation Kit (EMD Millipore) was used for immunoprecipitation of RNA-binding proteins. Cell lysate was prepared by $200 \mu \mathrm{l}$ RIP lysis Buffer, $1 \mu$ l Protease Inhibitor Cocktail and $0.5 \mu$ l RNase inhibitor. Magnetic bead tubes were vortexed for $30 \mathrm{sec}$ for full suspension; $50 \mu 1$ magnetic bead suspension, $100 \mu 1 \mathrm{RIP}$ Wash Buffer heavy suspension beads, $5 \mu \mathrm{g}$ Ago 2 antibody (cat. no. 2897; Cell Signaling Technology, Inc., Danvers, MA, USA) was added to clean the beads twice. Immunoprecipitation of RNA-binding Protein-RNA complexes was performed. The magnetic beads and cell lysate were centrifuged at $4^{\circ} \mathrm{C}$, $21,480 \mathrm{x} g$ for $10 \mathrm{~min}$. The beads were added to the magnetic beads tube and incubated overnight on the suspension apparatus at $4^{\circ} \mathrm{C}$. RNA was purified and precipitated using $80 \%$ ethanol. The supernatant was discarded by centrifugation at $21,480 \mathrm{x} \mathrm{g}$ for $15 \mathrm{~min}$ and then dried. RNA was dissolved in 10-20 $\mu \mathrm{l}$ RNase-free water, placed on ice and the RT-PCR was performed using the TaqMan ${ }^{\circledR}$ MicroRNA Reverse Transcription Kit, with all operations performed according to the manufacturer's instructions. The contents of LINC00887 and levels of miR-613 (forward, 5'-CCGCTCGAGTCTACT AGGTGTGGGCTTTA-3'; reverse, 3'-GGCAAGCTTCTG TGGCCTTCCTTACTCTT-5'); miR-206 (forward, 5'-TGG AATGTAAGGAAGTGTGTGG-3'; reverse, 3'-ACCTGACCG GCCGTAACAACCC-5') and miR-1-2 (forward, 5'-UGUAUG AAGAAAUGUAGGUAU-3'; reverse, 3'-ACAUACUUCUUU ACACCCAUA-5') were detected by conventional qPCR using TaqMan probes. TaqMan ${ }^{\circledR} 2 \mathrm{X}$ Universal PCR Master Mix was used under the following conditions: Initial denaturation at $95^{\circ} \mathrm{C}$, for $10 \mathrm{~min}$ followed by 40 cycles of $95^{\circ} \mathrm{C}$ for $15 \mathrm{sec}, 60^{\circ} \mathrm{C}$ for $1 \mathrm{~min}$ and $72^{\circ} \mathrm{C}$ for $45 \mathrm{sec}$. The level of LINC00887 was examined by using qPCR with $\mathrm{X}$ inactive specific transcript (XIST) as the negative control (forward, 5'-CTTGGATGG TTGGTTGCCAGCTA-3'; reverse, 3'-TCATGCCATCCA CCTA-5'); U6 RNA (forward, 5'-TGCTCGCTTCGGCAG C-3'; reverse, 3'-ACTACATGTGCATGCT-5') was used as the internal reference for miRNA. TaqMan probe was utilized to detect the level of miR-613, miR-206 and miR-1-2, with the U6b promoters the negative control.

Prediction and expression regulation of miRNA downstream targeting genes. The downstream interacting genes of miR-613, miR-206 and miR-1-2 were predicted using microRNA.org (microrna.org/microrna/getMirnaForm.do) and screening the mutually interacting genes fibronectin 1 (FN1), MET proto-oncogene, receptor tyrosine kinase (MET) and SMAD4 (according to the mirSVR score <-1.0). Then, LINC00887 was overexpressed in 95-C cell lines, and knocked down in 95-D cell lines using siRNA2. Changes of gene expression products of three genes were analysed by qPCR. TB Green Advantage qPCR premixes (Takara Biotechnology Co., Ltd.) was used in qPCR for these genes. qPCR was performed using the 7500 fast system with as previously described above. The primers of these were as follows: MET, forward, 5'-TCCTCTGGGAGC TGATGACA-3' and reverse, 3'-CTGGGCAGTATTCGGGTT GT-5'; FN1, forward, 5'-AGCCTGGGAGCTCTATTCCA-3' 
and reverse, 3'-CTTGGTCGTACACCCAGCTT-5'; SMAD4, forward, 5'-GTCAGCTGCTGCTGGAATTG-3' and reverse 3'-GTCTTGGGTAATCCGGTCCC-5'. $\beta$-actin was used as the internal reference. Accordingly, changes in the genomic products of FN1, MET and SMAD4 were observed.

Statistical analysis. SPSS statistical software (version 18.0; SPSS, Inc., Chicago, IL, USA) used to perform statistical analysis. Data are expressed as the mean \pm standard deviation from at least three experiments. Statistical comparisons were based on the Student's t-test or one-way analysis of variance (ANOVA). The Student' s t-test was used to compare the differences between two groups of mean values. The ANOVA test followed by a post-hoc test (least significance difference test, Student-Newman-Keuls) was used for evaluating the difference among multiple groups. $\mathrm{P}<0.05$ was considered to indicate a statistically significant difference.

\section{Results}

Association between LINC00887 and clinical overall survival time. RT-qPCR validation results suggested that LINC00887 is highly expressed in 95-D cells. In a sample containing of 522 cases of adenocarcinoma of the lung in the eBioPortal database (cbioportal.org), LINC00887 was overexpressed in $11 \%$ ( $\mathrm{Z}$ score \pm 1$)$ and the survival time of the patient in these cases was significantly lower than in patients without LINC overexpression $(\mathrm{P}=0.0141)$. In another dataset containing of 504 cases of lung carcinoma, LINC00887 gene expression was found in $38 \%$ of the cases. Although only $9 \%$ had an overexpressed LINC00887 level ( $Z$ score \pm 1$)$, the survival time of patient was also significantly reduced in these patients, which implies the potential importance this LINC00887 in the development of lung cancer.

In-cell localization of LINC00887. The results of LSCM observation revealed that $18 \mathrm{~S}$ RNA was present in the cytoplasm of 95-D and 95-C cells (Fig. 1). Therefore, LINC00887 was also located in the cytoplasm, with almost no expression found in the cell nuclei of 95-D cells. The LINC00887 expression level in 95-D cells was far higher than in 95-C cells.

LINC00887 influences the cell cycle and apoptosis of lung cancer cell lines. LINC00887 was transfected and overexpressed in 95-C cells and reduced by siRNA 95-D cells, and MTT assay results showed only a small difference, but no statistical significance in these two groups when the cells were cultured for 24 and $72 \mathrm{~h}$. It is suggested that LINC00887 has no significant effect on the proliferation of 95-C and 95-D cells (Fig. 2A). Meanwhile, in the overexpressed group of LINC00887 in 95-C cells, the LINC00887 RNA level was significantly higher than that of the control group, indicating that the upregulation was successful and effective (Fig. 2B). The 95-C/95-D cells were prepared using Annexin-FITC and Annexin-PI reagent kits. The distribution and proportion of cells in stage of the cell cycle was detected via flow cytometry, and the number of apoptotic cells was calculated. Apoptosis analysis showed that increase in LINC00887 expression reduced apoptosis of 95-C cells, while removing LINC00887 knockdown induced a higher

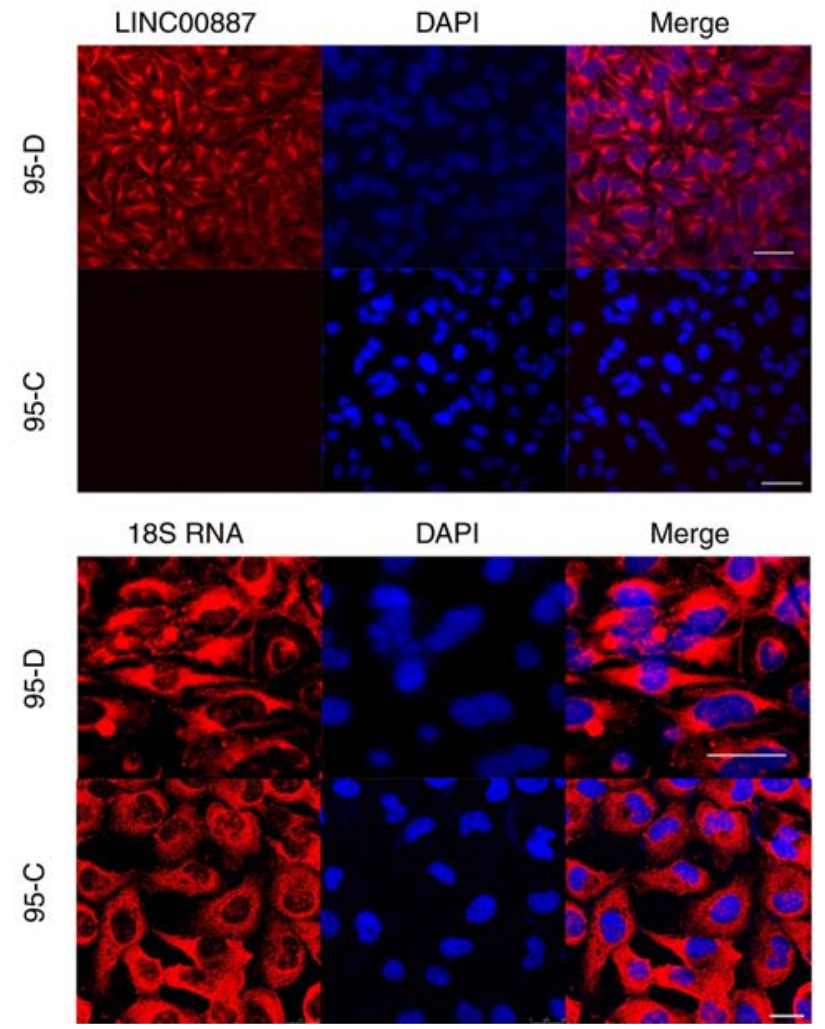

Figure 1. Cellular location of LINC00887 identified by laser scanning confocal microscopy observation. LINC00887, long intergenic non-protein coding RNA 887

apoptosis rate in 95-D cells (Fig. 2C). No difference detected between the G2/M, S and G1 phases of the cycle after flow cytometry examination (Fig. 2D). Furthermore, Transwell assays revealed that LINC00887 overexpression enhances the invasion of lung cancer cells (Fig. 2E). When LINC00887 was silenced from 95-D cells, the spheroidisation ability of 95-D cells was significantly reduced (Fig. 2F).

Interaction between LINC00887and genes in the TGF- $\beta$ signal transduction pathway. On the basis of bioinformatics analysis of the Ensembl database, enhancers are present in the binding sequence (TGAC/GTCA) of a TPA-responsive element and in the binding motif sequence (GTCT/AGAC) of a SMAD binding element. Two plasmids of pcDNA3.1-LINC00887 and pGL3 basic-enhancer, which was contained the LINC00887 sequence and the luciferase, were generated for use in reporter system. Restriction enzyme analysis was performed, and the plasmids were expressed on $796 \mathrm{nt}$ and $504 \mathrm{nt}$ bands on the PCR adhesive strip and the results as shown in (Fig. 3A). The transcriptional activity of LINC00887 in 95-D cells with added TGF- $\beta$ was significantly increased, and the relative luciferase activity was significantly increased compared with the control 95-D cell group ( $\mathrm{P}=0.0004$; Fig. 3B). When ITD-1 was added to the cells, the TGF- $\beta$ pathway was suppressed and the relative luciferase activity associated with LINC00887 expression was significantly reduced, (TGF + ITD-1 vs. TGF- $\beta ; \mathrm{P}=0.0002$; Fig. 3B).

miR-613, miR-206 and miR-1-2 are ceRNAs associated with LINC00887. The online searching results for LINC00887 


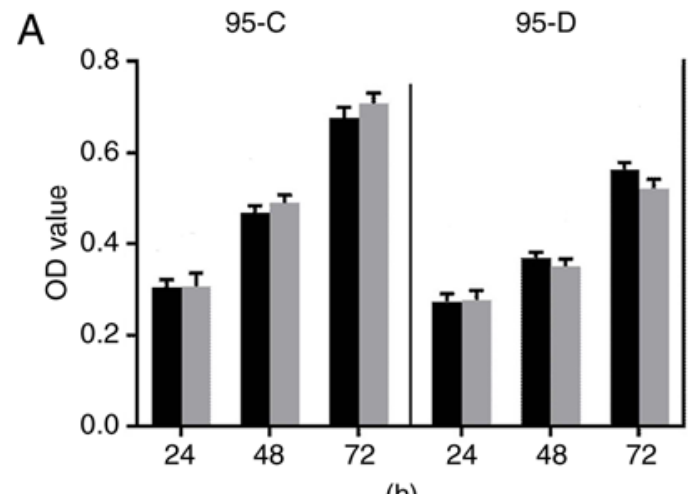

C

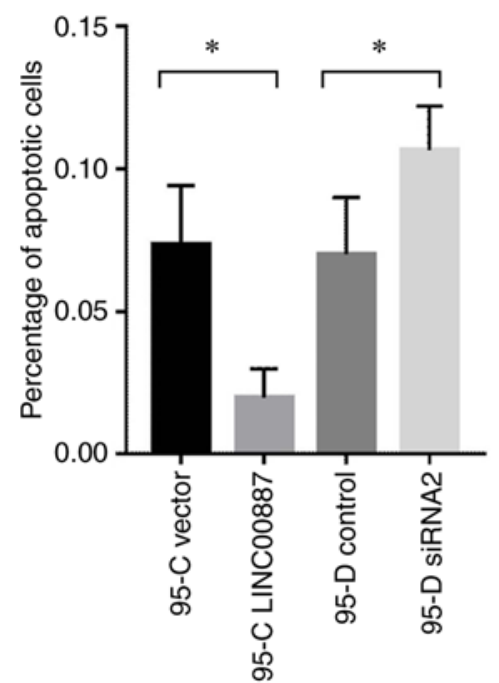

E

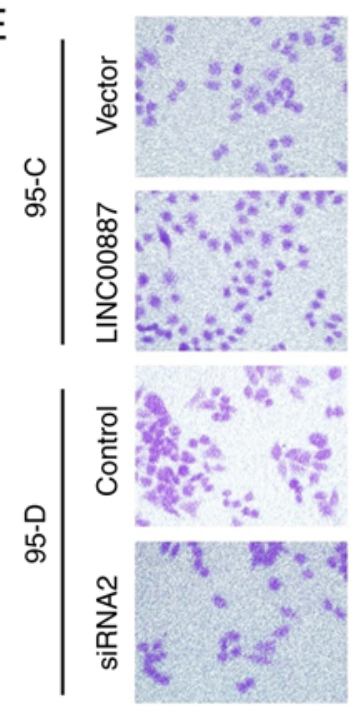

Control

LINC00887/siRNA2
B
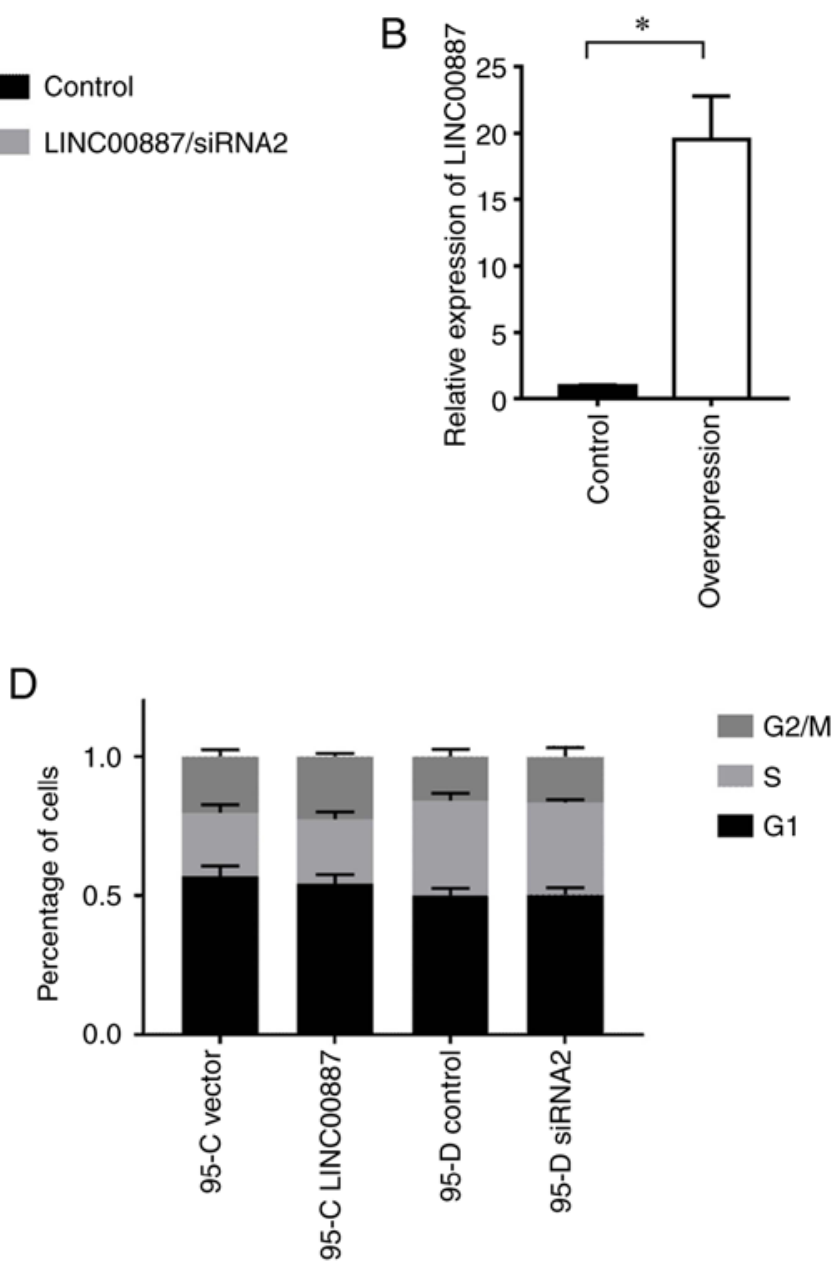

Figure 2. Effects of LINC00887 on the metastasis, apoptosis and stem cell characteristic transformation of 95-C/95-D cells. (A) Knockdown and overexpression of LINC00887. A small difference was found in the 95-C and 95-D cells at 24 and 72 h, but no statistical significance in the MTT assay. (B) LINC00887 was overexpressed in 95-C cells using the overexpression vector. (C) Increased LINC00887 expression can reduce apoptosis of 95-C cells; while knockdown it could induce a higher apoptosis rate in 95-D cells. (D) No obvious difference was found in G2/M, S and G1 phase proportions. (E) Transwell assays found that LINC00887 overexpression can enhance the invasion of lung cancer cells and stem cell characteristics. (F) Spheroidisation ability of 95-D cells with LINC00887 siRNA. " $\mathrm{P}<0.05 ;{ }^{* * * *} \mathrm{P}<0.001$. OD, optical density; siRNA, small interfering RNA; LINC00887, long intergenic non-protein coding RNA 887.

(http://www.lncipedia.org/db/search) indicated that the five top rated miRNAs were miR-1227, miR-613, miR-206, miR-5581-3p and miR-1-2. The base pairing ability of each miRNA to LINC00887 was evaluated as 77.67, 73.48, 73.29, 67.50 and 65.30 , respectively. It was noted that miR-613,
miR-206 and miR-1-2 have similar downstream target genes. Simulated hybridization suggested that the seed sequences in these targeting miRNAs were fully integrated with LINC00887, as shown in Fig. 4A. When pGL3-LINC00887 or empty vector were co-transfected into $293 \mathrm{~T}$ cells with miRNA 
A

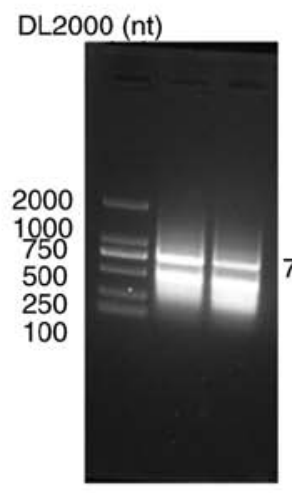

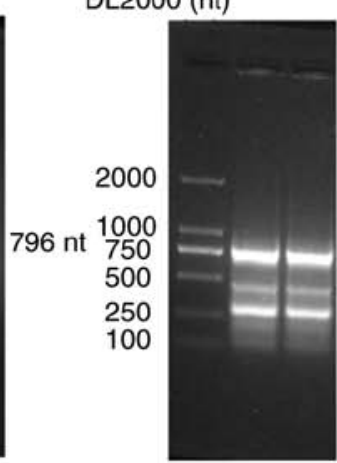
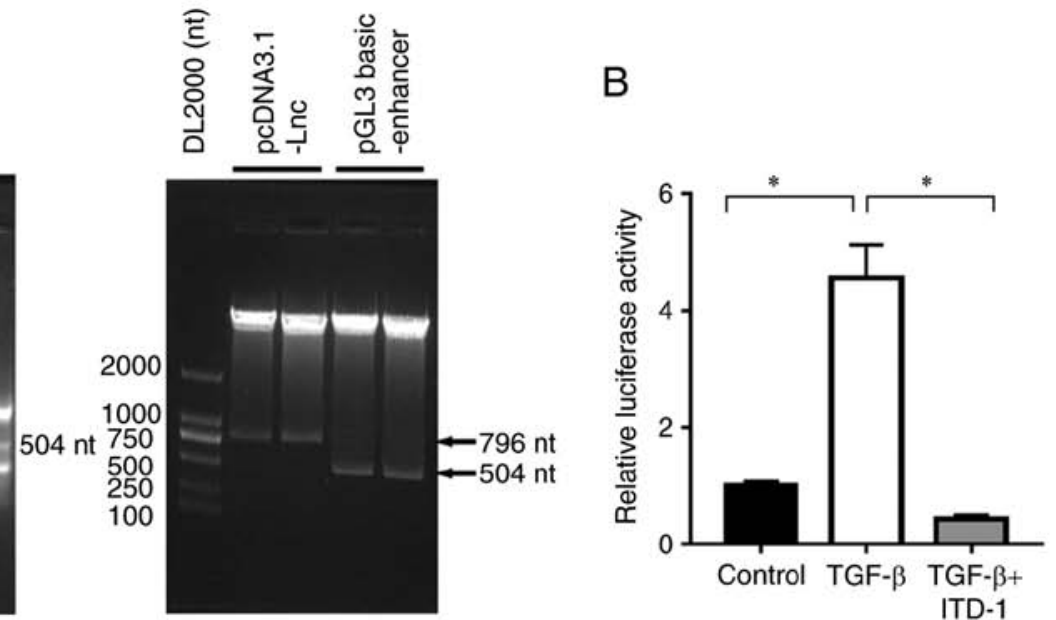

Figure 3. LINC00887 may promote cell metastasis through TGF- $\beta$ signalling pathway in $95-\mathrm{D}$ cells. (A) Plasmid $796 \mathrm{nt}$ and 504 nt bands separated on agarose gel; (B) transcriptional activity of LINC00887 in 95-D cells treated with TGF- $\beta$ and ITD-1. * P $<0.05$. LINC00887, long intergenic non-protein coding RNA 887; TGF- $\beta$, transforming growth factor- $\beta$; ITD-1, selective inhibitor of TGF- $\beta$ signalling.

A

5iR-613

miR-206 3' gguguguGAAGGAAuguaaggu 5'

5'

miR-1-2 $\quad 3{ }^{\prime}$ uauguaugaagaaauGUAGGU $5^{\prime}$

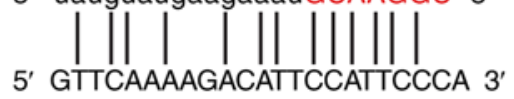

B

Vector

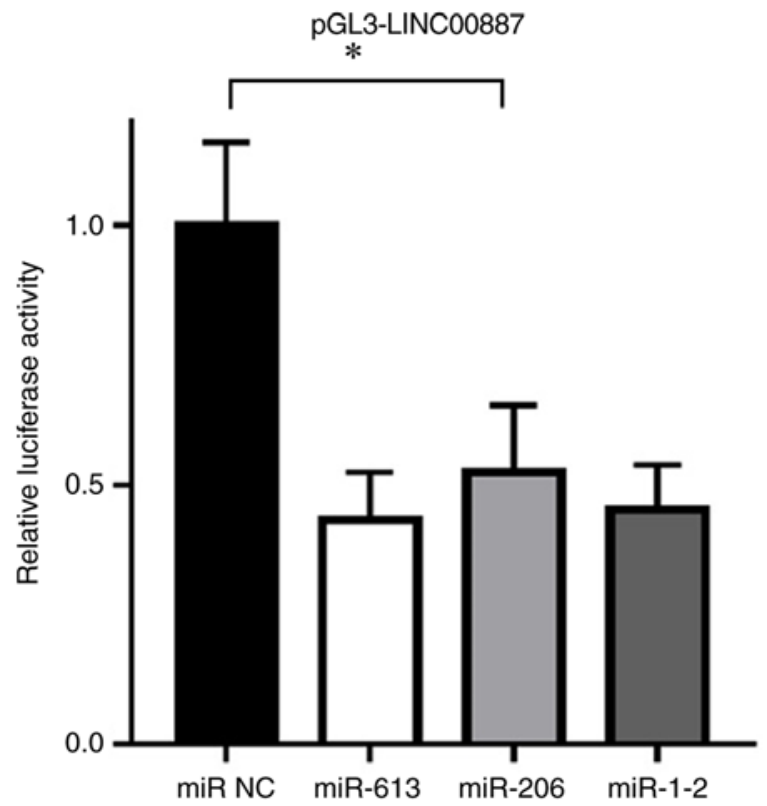

Figure 4. Luciferase reporting system results suggest that LINC00887 can effectively bind to three miRs. (A) Simulated hybridization of the seed sequences (marked in red) in three miRs were fully integrated with LINC00887. (B) Effect of miRs on luciferase activity of empty vector (P $>0.05)$ and activity of luciferase in pGL3-LINC00887-transfected 293T cells ( $\mathrm{P}<0.05)$. miR, microRNA; LINC00887, long intergenic non-protein coding RNA 887.

precursors for $24 \mathrm{~h}$ and luciferase activity was tested, the miRNA precursors had no effect on the luciferase activity in the empty vector group. However, these miRNAs suppressed the luciferase activity of pGL3-LINC00887. Therefore, it can be concluded that miR-613, miR-206 and miR-1-2 bind with LINC00887 act as ceRNAs (Fig. 4B). 

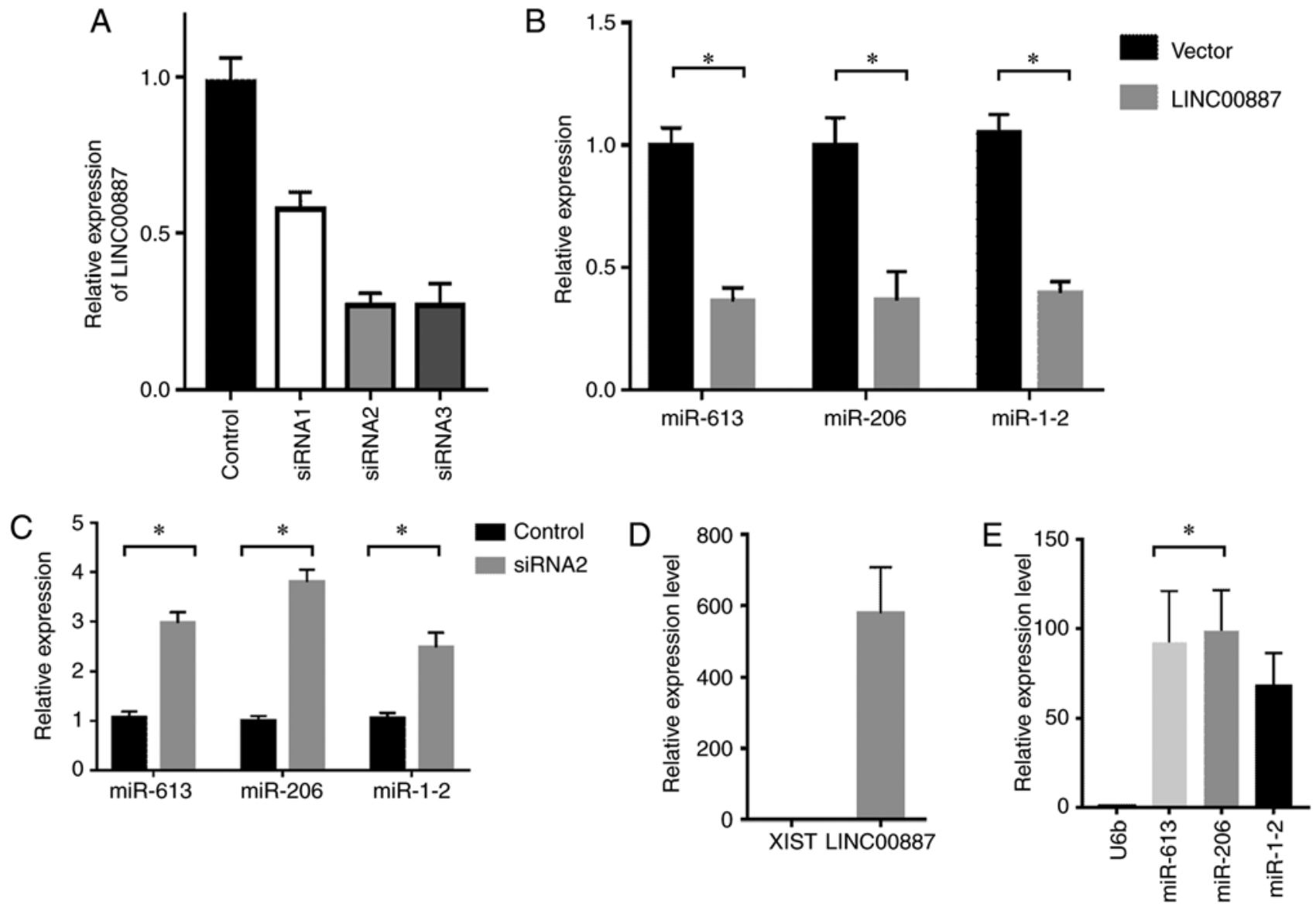

Figure 5. LINC00887 and three miRNAs formed competitive inhibitory effects. (A) By synthesizing three interference sections of LINC00887 siRNA2 had the best interference results in 95-D cells. (B) In 95-D cells with overexpressed LINC00887, the levels of three miRs were reduced. (C) Knockdown of LINC00887 by siRNA2, indicated that it can increased the expression of the three miRs in 95-C cells. Immunoprecipitation with Ago2 antibody and reverse transcription-quantitative polymerase chain reaction were used to show that (D) LINC00887 and (E) the associated miRs interacted with Ago2, indicating that degradation was triggered after the absorption of miRs. ${ }^{~} \mathrm{P}<0.05$. LINC00887, long intergenic non-protein coding RNA 887; siRNA, small interfering RNA; miR, microRNA; XIST, $X$ inactive specific transcript.

LINC00887 can combine with miRNAs in 95-C/95-D cells resulting in RNA degradation. By synthesizing three interference sections of LINC00887 (Guangzhou RiboBio Co., Ltd.), i.e. antagomir 1, 2 and 3, it was found that siRNA2 had the best interference results in 95-D cells (Fig. 5A). Empty vector and LINC00887 overexpression vector were transfected into 95-C cells, and the expression levels of miRNAs were analysed using a TaqMan probe. The results indicated LINC00887 overexpression reduced the levels of miR-613, miR-206 and miR-1-2 (Fig. 5B). This suggested a linear association between the miRNAs and LINC00887.

Similarly, these miRNAs were increased in 95-D cell lines when the expression of LINC00887 was silenced using by siRNA (Fig. 5C). Immunoprecipitation was performed out using an Ago2 antibody; the nucleic acid content of the products bound to Ago2 was subsequently purified and quantified. The levels of XIST (negative control) and LINC00887 associated with Ago2 were analysed by RT-qPCR. The internal reference gene U6b (negative control) and the levels of miR-613, miR-206 and miR-1-2 were also analysed using TaqMan probes. The results suggested that LINC00887 and its associated miRNAs interacted with Ago2, indicating that degradation was triggered by the binding with miRNAs (Fig. 5D and E).
LINC00887 and miRNA hybrids degrade and inactivate the downstream targeting genes FN1, MET and SMAD4. Using a mirSVR score of $<-1.0$ as the criterion, FN1, MET and SMAD4 were ranked as the top three downstream genes targeted by miR-613, miR-206 and miR-1-2 in the microRNA. org database (Fig. 6A). When LINC00887 was overexpressed in 95-C cell lines the mRNA levels FN1, MET and SMAD4 significantly increased compared with the control group (empty vector). This indicated that LINC00887 effectively combines with and degrade miR-613, miR-206 and miR-1-2s, and therefore suppresses their actions on the downstream target genes (Fig. 6B). Similarly, in 95-D cell lines with initial high expression of LINC00887, FN1, MET and SMAD4 were reduced by LINC00887 siRNA ( $\mathrm{P}<0.0001$; Fig. 6C).

\section{Discussion}

Although the function of most lncRNAs remains unknown thus far, it is of great interest to find out whether they have could be used for diagnosing and treating clinic diseases. In the current study, 95-D and 95-C cells, with different expression levels of LINC00887, were used to investigate the role of LINC00887. According to the data from eBioPortal, patients with a high level of LINC00887 generally have shorter overall 
A

miR-613

3' ccGUUUCUUCCUUGUAAGGa 5' || | | |||||| 671:5' aaUAAAAGAUUUACAUUCCa $3^{\prime}$

$\operatorname{miR}-206$

miR-1-2

| | | | | | | | |

3' uauguaugaagAAAUGUAAGGu $5^{\prime}$ |l||l||l|| 669-5' uuaauaaaagaUUUACAUUCCa 3'

SMAD4

miR-613

miR-1-2
3' ccguUUCUUCCUU-GUAAGGa 5' | |||||||| |||||| 258:5' auguAUGAAGGAAUCAUUCCa $5^{\prime}$

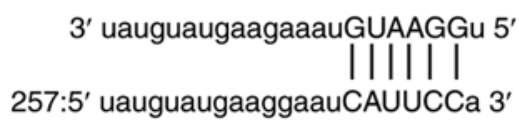

MET

miR-613 3' ccguuucUCC- -UUGUAAGGa 5'<smiles>C#CC(=C)C(=C)C(=C)C(=C)C=C</smiles>

485:5' cacccauuAGGUAAACAUUCCc 3'

miR-206 3' ggUGUGUGAAGGA-AUGUAAGGu 5'

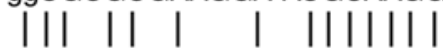

484:5' ucACCCAUUAGGUAAACAUUCCc 3

miR-1-2 3' uauguaugaagaaaUGUAAGGu 5'

|| || || |

485:5' cacccauuagguaaACAUUCCc $3^{\prime}$
B

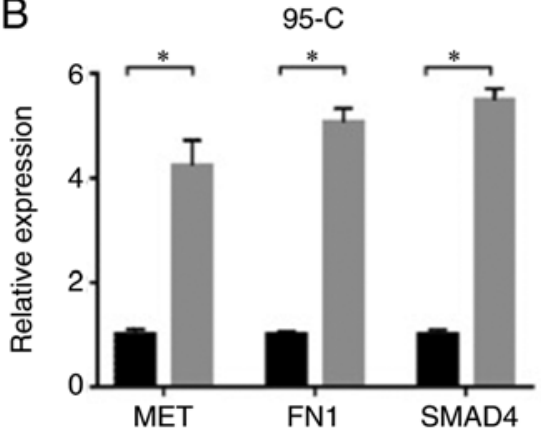

C

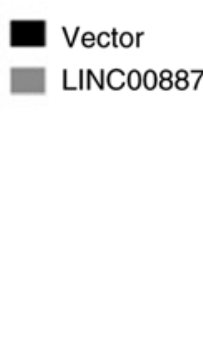

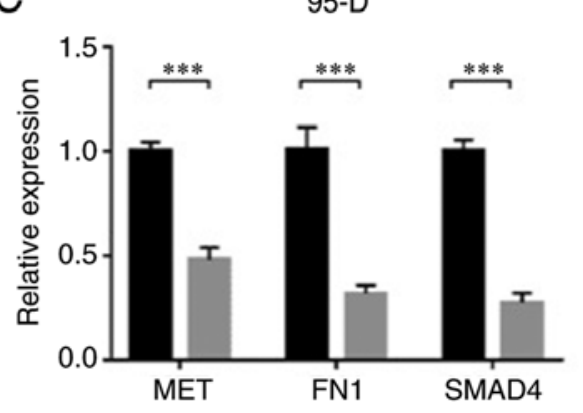

Control siRNA2

Figure 6. LINC00887 can regulate downstream genes MET, FN1 and SMAD4. (A) LINC00887 and the three miRNAs interact with each other. In 95-C cell lines, LINC00887 can effectively combine and degrade these miRNAs, and therefore suppress and regulate the downstream genes (B) Overexpression of LINC00887 increased MET, FN1 and SMAD4 levels ( $\left.{ }^{*} \mathrm{P}<0.05\right)$. (C) LINC00887 siRNA reduced the expression of MET, FN1 and SMAD4 (t-test, $\left.{ }^{* * *} \mathrm{P}<0.0001\right)$. miR, microRNA; MET, MET proto-oncogene, receptor tyrosine kinase; FN1, fibronectin 1; mothers against decapentaplegic homolog 4; LINC00887, long intergenic non-protein coding RNA 887.

survival time, indicating LINC00887 has specific functions and clinical value for patients with NSCLC. High levels of LINC00887 may potentially accelerate the malignant evolution of NSCLC cells, and therefore, induce the metastasis and spread of tumor cells to the rest of the body. In addition, Transwell experimental results suggested that LINC00887 significantly enhanced the invasion of lung cancer cells. By silencing LINC00887 in 95-D cells, their spheroidisation ability was significantly reduced, which indicates a reduced potential for spreading and survival at other sites. These results suggest that LINC00887 is a persistent and functional IncRNA.

From the results of the current study, it can be concluded that LINC00887 exerts its function by interacting with miRNAs, and these interact as ceRNAs (21). The ceRNA theory suggests that downstream targeting gene functions are affected by changes in miRNA quantity induced by bas pairing between lncRNAs and miRNAs. ceRNAs generally include miRNAs, pseudogene transcripts and circular RNAs (22). The most common way for lncRNAs to interact with miRNAs is through a so-called RNA sponge effect, which is determined by stable expression of lncRNAs and miRNAs and their integration with miRNA response elements (MRE) (23). In a study of gallbladder carcinoma (24), lncRNA MATLAT1 acted as a ceRNA regulating miR-206 through a molecular sponge mechanism and promoted the development of cancer. In this study, LINC00887 was identified in the cytoplasm only and expressed highly in nuclei.

Hybridization simulation suggested many miRNAs can interact with LINC00887, among which miR-613, miR-206 and miR-1-2 were ranked as the top three candidates. When LINC00887 was silenced, the levels of miR-613, miR-206 and miR-1-2 increased significantly; however, when LINC00887 was overexpressed in 95-C cells, the levels of miR-613, miR-206 and miR-1-2 were significantly reduced, indicating an inversely proportional relationship between LINC00887 and certain miRNAs. It remains unclear if the association between LINC00887 and miR-613, miR-206 and miR-1-2 temporarily suppresses their activity or induced RNA degradation. By examining the RNA pulldown products isolated by Ago2 
immunoprecipitation, it was found that the concentrations of LINC00887, miR-613, miR-206 and miR-1-2 were all higher than the negative control RNAs (XIST and U6b), indicating that interaction between the LINC00887 can induced degradation of miRNAs. These experiments suggested that there is a linear relationship between LINC00887 and miR-613, miR-206 and miR-1-2, and that binding with LINC00887 can induce the degradation of miRNAs by Ago2. Therefore, miR-613, miR-206 and miR-1-2 can act as ceRNAs of LINC00887.

The metastasis of lung cancer is a multifaceted process (25). Metastasis involves the migration of lung cancer cells towards areas of higher oxygen tension and changing of cellular phenotype through EMT, changes in the expression of vascular endothelial growth factor (VEGF) and its receptors (26), stroma interaction (27) and interfering with immune system communication (28). Recent studies have shown that IncRNAs can control protein transcription, translation and function. It is now believed that dozens of lncRNAs are involved in lung cancer cell invasion and metastasis $(29,30)$.

In this study, it was demonstrated that LINC00887-miRNA interaction regulates downstream genes including FN1, MET and SMAD4, which are associated with the spread of lung cancer. The results suggested that overexpression of LINC00887 in 95-C cell lines can reduce the expression of miR-613, miR-206 and miR-1-2. In contrast, FN1, MET and SMAD4 mRNA expression was increased by overexpression of LINC00887, which indicated an interaction between LINC00887 and three associated miRNAs and that this induced their degradation. The regulatory effects on downstream genes are therefore released, which potentially increases the motility and invasion of 95-C cells.

FN1 is a component of the extracellular matrix (ECM) and is widely distributed in smooth muscle cell layers (31). Abnormal expression of FN1 has been identified in several human diseases, including cancers, and can act as an important marker of EMT (32). miR-206 is involved in the formation of smooth muscle in the airway and is reported to be associated with FN1, interact, with ECM proteins such as VEGF and TGF- $\beta 1$, and contribute to the development of diseases including bronchopulmonary dysplasia (33). Relevant studies on miR-1 and MET genes revealed that miR-1 inhibited cell proliferation, and reduced migration and motility of A549 cells. The downregulation of MET could be a potential mechanism by which miR-1 regulates the growth and metastatic potential of these cells (34). SMAD4 is a key regulator of TGF- $\beta$ signalling. SMAD4 not activates the expression and increases the activity of EMT transcription factors (35-37).

Therefore, interactions between LINC00887-miRNAs and regulatory target genes may have an important roles in the invasion and metastasis of lung cancer cells; LINC00887-miRNA associations can affect NSCLC cell EMT by altering the TGF- $\beta$ signalling pathway. In this study, the transcription of LINC00887 was significantly increased by adding TGF- $\beta$ to 95-D cells, this effect was suppressed by the specific TGF- $\beta$ pathway inhibitor, ITD-1. The cell cycle and proliferation of 95-C cells was unaffected by overexpression and silencing of LINC00887; however, cell apoptosis was altered by changes in LINC00887 expression. Of course, in vivo experiments are required to further validate the role of LINC00887. We plan to use a lentivirus packaging vector to knock out the expression of LINC00887 in 95-D cells, and inoculate the cell line into nude mice to observe the growth of tumours, and detect the changes in associated metastasis indicators in metastatic tumours.

In summary, although the mechanism of NSCLC metastasis is complex, the present study established an interactive association between LINC00887 and miR-613, miR-206 and miR-1-2, providing a new path for studying metastasis mechanisms. LINC00887, and miR-613, miR-206 and miR-1-2 act as ceRNAs, and potentially regulate the EMT transition of NSCLC cells through the TGF- $\beta$ pathway, therefore promoting cell migration and the acquisition of stem cell features. It should be noted that this study is a preliminary investigation into the function of LINC00887, particularly with respect to signal transduction pathways. EMT mechanisms via TGF- $\beta$ signalling are complex, and further study is required.

\section{Acknowledgements}

Not applicable.

\section{Funding}

This study was funded by The Program for Research Projects of the Shaanxi Provincial Health Department (grant no. 2016D036), Shaanxi Provincial Traditional Chinese Medicine Administration Chinese Medicine Research Project (grant no. JCPT039), Research for Projects of the Xi'an technology office program (grant no. 2016047SF/YX03(4), Key Projects of Social Development Research Programs of Shaanxi Province (grant no. 2017SF-199), Natural Science Basic Research Program of Shaanxi Province (grant no. 2017JM8195, 2018JM7044) and National Natural Science Foundation (grant no. 81672300).

\section{Availability of data and materials}

The datasets used and/or analysed during the current study are available from the corresponding author on reasonable request.

\section{Authors' contributions}

YT and SY made substantial contributions to conception and design. YT, MY, LS and SH performed acquisition of data. SS, WS and JW analysed and interpreted data. LL, QH, YD and JZ have been involved in drafting the manuscript or revising it critically for important intellectual content. SS and XR contributed to the checking of article data and the calculation of statistical methods. JS provided experimental technical support, and checked the integrity of the data. All authors have read and approved the final manuscript.

\section{Ethics approval and consent to participate}

Not applicable.

\section{Patient consent for publication}

Not applicable.

\section{Competing interests}

The authors declare that they have no competing interests. 


\section{References}

1. Torre LA, Bray F, Siegel RL, Ferlay J, Lortet-Tieulent J and Jemal A: Global cancer statistics, 2012. CA Cancer J Clin 65: 87-108, 2015.

2. Hashim D, Boffetta P, La Vecchia C, Rota M, Bertuccio P, Malvezzi $M$ and Negri E: The global decrease in cancer mortality: Trends and disparities. Ann Oncol 27: 926-933, 2016.

3. Wang L, Yu C, Liu Y, Wang J, Li C, Wang Q, Wang P, Wu S and Zhang ZJ: Lung cancer mortality trends in China from 1988 to 2013: New challenges and opportunities for the government. Int J Environ Res Public Health 13: E1052, 2016.

4. Stratton MR, Campbell PJ and Futreal PA: The cancer genome. Nature 458: 719-724, 2009.

5. Ansari J, Yun JW, Kompelli AR, Moufarrej YE, Alexander JS, Herrera GA, Herrera GA and Shackelford RE: The liquid biopsy in lung cancer. Genes Cancer 7: 355-367, 2016.

6. MacConaill LE and Garraway LA: Clinical implications of the cancer genome. J Clin Oncol 28: 5219-5228, 2010.

7. Ponting CP, Oliver PL and Reik W: Evolution and functions of long noncoding RNAs. Cell 136: 629-641, 2009.

8. Tsai MC, Manor O, Wan Y, Mosammaparast N, Wang JK, Lan F, Shi Y, Segal E and Chang HY: Long noncoding RNA as modular scaffold of histone modification complexes. Science 329: 689-693, 2010.

9. Prensner JR, Iyer MK, Balbin OA, Dhanasekaran SM, Cao Q, Brenner JC, Laxman B, Asangani IA, Grasso CS, Kominsky HD, et al: Transcriptome sequencing across a prostate cancer cohort identifies PCAT-1, an unannotated lincRNA implicated in disease progression. Nat Biotechnol 29: 742-749, 2011.

10. Gibb EA, Brown CJ and Lam WL: The functional role of long non-coding RNA in human carcinomas. Mol Cancer 10: 38, 2011.

11. Braconi C, Valeri N, Kogure T, Gasparini P, Huang N, Nuovo GJ, Terracciano L, Croce CM and Patel T: Expression and functional role of a transcribed noncoding RNA with an ultraconserved element in hepatocellular carcinoma. Proc Natl Acad Sci USA 108: 786-791, 2011

12. Mourtada-Maarabouni M, Pickard MR, Hedge VL, Farzaneh F and Williams GT: GAS5, a non-protein-coding RNA, controls apoptosis and is downregulated in breast cancer. Oncogene 28 : 195-208, 2009.

13. Huarte M, Guttman M, Feldser D, Garber M, Koziol MJ Kenzelmann-Broz D, Khalil AM, Zuk O, Amit I, Rabani M, et al: A large intergenic noncoding RNA induced by 153 mediates global gene repression in the p53 response. Cell 142: 409-419, 2010.

14. Zhang X, Gejman R, Mahta A, Zhong Y, Rice KA, Zhou Y, Cheunsuchon P, Louis DN and Klibanski A: Maternally expressed gene 3, an imprinted noncoding RNA gene, is associated with meningioma pathogenesis and progression. Cancer Res 70: 2350-2358, 2010.

15. Poliseno L, Salmena L, Zhang J, Carver B, Haveman WJ and Pandolfi PP: A coding-independent function of gene and pseudogene mRNAs regulates tumour biology. Nature 465: 1033-1038, 2010.

16. Hao Y, Yang X, Zhang D, Luo J and Chen R: Long noncoding RNA LINC01186, regulated by TGF- $\beta$ /SMAD3, inhibits migration and invasion through Epithelial-Mesenchymal-Transition in lung cancer. Gene 608: 1-12, 2017.

17. Yang $\mathrm{C}, \mathrm{Li} \mathrm{X}$, Wang $\mathrm{Y}$, Zhao $\mathrm{L}$ and Chen W: Long non-coding RNA UCA1 regulated cell cycle distribution via CREB through PI3-K dependent pathway in bladder carcinoma cells. Gene 496: 8-16, 2012.

18. Wang JZ, Xiang JJ, Wu LG, Bai YS, Chen ZW, Yin XQ, Wang Q, Guo WH, Peng Y, Guo $\mathrm{H}$ and $\mathrm{Xu}$ P: A genetic variant in long non-coding RNA MALAT1 associated with survival outcome among patients with advanced lung adenocarcinoma: A survival cohort analysis. BMC Cancer 17: 167, 2017.

19. Salmena L, Poliseno L, Tay Y, Kats L and Pandolfi PP: A ceRNA Hypothesis: The Rosetta stone of a hidden RNA language? Cell 146: 353-358, 2011.
20. Livak KJ and Schmittgen TD: Analysis of relative gene expression data using real-time quantitative PCR and the 2(-Delta Delta C(T)) method. Methods 25: 402-408, 2001.

21. Thomson DW and Dinger ME: Endogenous microRNA sponges: Evidence and controversy. Nat Rev Genet 17: 272-283, 2016.

22. Venables JP, Klinck R, Koh C, Gervais-Bird J, Bramard A, Inkel L, Durand M, Couture S, Froehlich U, Lapointe E, et al: Cancer-associated regulation of alternative splicing. Nat Struct Mol Biol 16: 670-676, 2009.

23. Wang SH, Zhang WJ, Wu XC, Zhang MD, Weng MZ, Zhou D, Wang JD and Quan ZW: Long non-coding RNA Malatl promotes gallbladder cancer development by acting as a molecular sponge to regulate miR-206. Oncotarget 7: 37857-37867, 2016.

24. Popper HH: Progression and metastasis of lung cancer. Cancer Metastasis Rev 35: 75-91, 2016.

25. Decaussin M, Sartelet H, Robert C, Moro D, Claraz C, Brambilla $\mathrm{C}$ and Brambilla E: Expression of vascular endothelial growth factor (VEGF) and its two receptors (VEGF-R1-Flt1 and VEGF-R2-Flk1/KDR) in non-small cell lung carcinomas (NSCLCs): Correlation with angiogenesis and survival. J Pathol 188: 369-377, 1999.

26. Suzuki K, Sun R, Origuchi M, Kanehira M, Takahata T, Itoh J, Umezawa A, Kijima H, Fukuda S and Saijo Y: Mesenchymal stromal cells promote tumor growth through the enhancement of neovascularization. Mol Med 17: 579-587, 2011.

27. Xiang R, Luo Y, Niethammer AG and Reisfeld RA: Oral DNA vaccines target the tumor vasculature and microenvironment and suppress tumor growth and metastasis. Immunol Rev 222: 117-128, 2008.

28. Jiang C, Li X, Zhao $\mathrm{H}$ and Liu H: Long non-coding RNAs: Potential new biomarkers for predicting tumor invasion and metastasis. Mol Cancer 15: 62, 2016.

29. Rokavec M, Horst D and Hermeking H: Cellular model of colon cancer progression reveals signatures of mRNAs, miRNA, lncRNAs, and epigenetic modifications associated with metastasis. Cancer Res 77: 1854-1867, 2017.

30. Zhang X, Xu J, Wang J, Gortner L, Zhang S, Wei X, Song J, Zhang Y, Li Q and Feng Z: Reduction of MicroRNA-206 contributes to the development of bronchopulmonary dysplasia through up-regulation of fibronectin 1. PLoS One 8: e74750, 2013.

31. Yen CY, Huang CY, Hou MF, Yang YH, Chang CH, Huang HW, Chen $\mathrm{CH}$ and Chang HW: Evaluating the performance of fibronectin 1 (FN1), integrin $\alpha 4 \beta 1$ (ITGA4), syndecan-2 (SDC2), and glycoprotein CD44 as the potential biomarkers of oral squamous cell carcinoma (OSCC). Biomarkers 18: 63-72, 2013.

32. Duan J, Zhang X, Zhang S, Hua S and Feng Z: miR-206 inhibits FN1 expression and proliferation and promotes apoptosis of rat type II alveolar epithelial cells. Exp Ther Med 13: 3203-3208, 2017.

33. Nasser MW, Datta J, Nuovo G, Kutay H, Motiwala T, Majumder S, Wang B, Suster S, Jacob ST and Ghoshal K: Downregulation of Micro-RNA-1 (miR-1) in lung cancer. Suppression of tumorigenic property of lung cancer cells and their sensitization to doxorubicin-induced apoptosis by miR-1. J Biol Chem 283: 33394-33405, 2008.

34. Grelet S, McShane A, Geslain R and Howe PH: Pleiotropic roles of non-coding RNAs in TGF- $\beta$-mediated epithelial-mesenchymal transition and their functions in tumor progression. Cancers (Basel) 9: pii: E75, 2017.

35. Lamouille S, Xu J and Derynck R: Molecular mechanisms of epithelial-mesenchymal transition. Nat Rev Mol Cell Biol 15: 178-196, 2014.

36. Moustakas A and Heldin CH: Mechanisms of TGF- $\beta$-induced epithelial-mesenchymal transition. J Clin Med 5: E63, 2016.

37. Lee JK, Joo KM, Lee J, Yoon Y and Nam DH: Targeting the epithelial to mesenchymal transition in glioblastoma: The emerging role of fMET signaling. Onco Targets Ther 7: 1933-1944, 2014. 